\title{
Effect of First-Order PMD Compensation on the Statistics of Pulse Broadening in a Fiber with Randomly Varying Birefringence
}

\author{
Daniel Mahgerefteh, Member, IEEE, and Curtis R. Menyuk, Fellow, IEEE
}

\begin{abstract}
Polarization mode dispersion in standard telecommunication fibers can be compensated to first order by using the concept of principal states of polarization. At the receiver the pulse is decomposed into the two waveforms polarized along the two principal states for the optical link and their delay is removed. We show by Monte Carlo simulation that compensation sharpens the probability distribution function of the pulse durations by a factor that decreases with increasing polarization dispersion.
\end{abstract}

Index Terms-Optical fiber communications, optical fiber dispersion, optical propagation, polarization, compensation.

\section{INTRODUCTION}

$\mathbf{T}$ THE DEMAND for bandwidth is requiring the transmission of higher bit rates in older installed fibers where polarization mode dispersion is a limiting factor. The small, randomly varying birefringence of standard telecommunication fibers tends to depolarize optical signals and randomize their phase. This frequency-dependent phase variation broadens an input optical pulse as it propagates through the fiber, leading to intersymbol interference. A key concept in analyzing polarization mode dispersion in fibers with randomly varying birefringence is the principal state [1]. For a given length of fiber, there are two orthogonal states of polarization at the input that yield polarization states that are independent of frequency to first order at the output. These orthogonal polarization states, called the input and output principal states of polarization, are a convenient basis set for analysis of arbitrarily polarized pulses. Poole and Wagner [1] have shown that the polarization dispersion of any optical pulse after propagation through a fiber can be described to first order in terms of a differential group delay between two pulses having the polarization of the output principal states.

One can use the concept of principal states to compensate for polarization dispersion to some extent. Hass et al. [2] have shown that dispersion can be reduced by transmitting or receiving a principal state of polarization. There are also several optical and electronic techniques for compensation in

Manuscript received October 15, 1998; revised December 11, 1998.

D. Mahgerefteh is with the Laboratory for Physical Sciences, College Park, MD 20740 USA. He also with the Department of Electrical Engineering, University of Maryland, College Park, MD 20740. C. R. Menyuk is with the Laboratory for Physical Sciences, College Park, MD 20740 USA. He is also with the Department of Computer Science and Electrical Engineering, University of Maryland Baltimore County, Baltimore MD 21250 USA.

Publisher Item Identifier S 1041-1135(99)01875-3. which the signal is broken up into the two principal states and the differential group delay between the two states compensated either electronically [3] or optically [4]-[5]. Because of the random nature of PMD in telecommunication fibers, it is important to know how the statistics of pulse broadening is changed by such compensators. Analytical calculations, which treat first and second order PMD, are only valid for low PMD values and are not adequate for the evaluation of compensators in a real system.

Here, we use Monte Carlo simulations to assess a differential group delay compensator and determine the degree of compensation as a function of polarization dispersion. We compare the distributions for the pulsewidth increase with and without compensation after signal propagation through a fiber with randomly varying birefringence. We show that the compensator sharpens the distribution of pulse durations by an amount that depends on the ratio of the fiber length to the PMD length, defined as $L_{\mathrm{PMD}}=\left(T / D_{\mathrm{PMD}}\right)^{2}$. Here $T$ is the initial root-mean-square (rms) pulse duration, where the initial pulse is single polarization and transform-limited, and $D_{\text {PMD }}$ in $\mathrm{ps} / \sqrt{\mathrm{km}}$ is the polarization dispersion of the fiber. Physically, $L_{\mathrm{PMD}}$ is the length scale on which a pulse will begin to spread significantly due to polarization dispersion. We evaluate the degree of compensation by considering the change in means and standard deviations of the distribution of pulsewidths as well as the effect on the tail of the distributions with and without the compensator. We find that compensation reduces the mean and standard deviation of pulse durations by a factor that decreases with increasing polarization dispersion.

Theory: Consider an arbitrarily polarized time-varying field, $\boldsymbol{E}_{a}(t)=E_{a}(t) \exp \left(i \omega_{0} t\right) \hat{\boldsymbol{e}}_{a}$, launched into a fiber of length $L$ with randomly varying birefringence. We assume that there is no chromatic dispersion, as occurs when the average dispersion is zero, and no polarization-dependent loss. The optical field at the output can be written as the sum of two waveforms

$$
\boldsymbol{E}_{b}(t)=\boldsymbol{E}_{+}(t)+E_{-}(t)
$$

where $\boldsymbol{E}_{+}(t)$ and $\boldsymbol{E}_{-}(t)$ are given by

$$
\begin{aligned}
\boldsymbol{E}_{ \pm}(t)= & \int_{-\infty}^{+\infty} c_{ \pm}(\omega) \tilde{E}_{a}(\omega) \\
& \cdot \exp (i \omega t) \exp \left[i \phi_{b \pm}(\omega)\right] \hat{\boldsymbol{e}}_{b \pm}(\omega) d \omega .
\end{aligned}
$$


Here, $\tilde{E}_{a}(\omega)$ is the Fourier transform of the input field, $\hat{\boldsymbol{e}}_{b \pm}(\omega)$ and $\phi_{b \pm}(\omega)$ are the output principal states of polarization and their corresponding phases, $c_{ \pm}(\omega)=\hat{\boldsymbol{e}}_{a} \cdot \hat{\boldsymbol{e}}_{a \pm}^{*}(\omega)$ are the projections of the input polarization on the input principal states, $\hat{e}_{a \pm}$, and $\omega$ is frequency.

To compensate for polarization dispersion to first order, the output pulse is decomposed into the two principal states at the central frequency, $\omega_{0}$, and the waveform of the faster moving principal state is delayed by $-\Delta \tau\left(\omega_{0}\right)$ relative to the waveform of the slower moving principal state so that the two overlap in time. Here, $\Delta \tau(\omega)$ is the differential group delay between the output principal states of polarization. The compensated field may be written as

$$
\begin{aligned}
\boldsymbol{E}_{c}(t)= & \int_{-\infty}^{+\infty} \tilde{E}_{a}(\omega) e^{i \omega t} \\
& \times\left\{\exp \left[i \phi_{b+}(\omega)-\Delta \tau\left(\omega_{0}\right) / 2\right] c_{+}(\omega) \hat{\boldsymbol{e}}_{b+}\left(\omega_{0}\right)\right. \\
& \left.+\exp \left[i \phi_{b-}(\omega)+\Delta \tau\left(\omega_{0}\right) / 2\right] c_{-}(\omega) \hat{\boldsymbol{e}}_{b-}\left(\omega_{0}\right)\right\} d \omega .
\end{aligned}
$$

The first-order frequency dependence of the phase factors cancels in (4), leading to a reduced polarization dispersion. We determined the effect of this operation on the pulsewidth after propagation through a length of fiber.

Numerical Model: We used the model of Wai and Menyuk [6] in which the fiber is a series of segments of length $l$ having fixed and a wavelength-independent difference in the index of refraction, $\Delta n$, induced by the birefringence with a randomly varying axis orientation angle, $\theta_{i}$. In this model, the rate of change of the orientation with distance along the fiber is a dimensionless random variable $g_{i}$ with zero mean. For an ensemble of fiber realizations to have a given $D_{\text {PMD }}$ independent of the length of the segments, $g_{i}$ has to have a standard deviation $\left\langle g_{i}^{2}\right\rangle^{1 / 2}=2 \Delta n / D_{\mathrm{PMD}} \times d^{1 / 2}$, where $D_{\mathrm{PMD}}=\sqrt{\left\langle\Delta \tau^{2}\right\rangle / L}, c$ is speed of light, and $\left\langle\Delta \tau^{2}\right\rangle^{1 / 2}$ is the ensemble average differential group delay.

For each fiber realization, we solved for the output Jones vector $\hat{\boldsymbol{e}}(z, \omega)$ and the dispersion vector $\Omega(z, \omega)$ defined on the Poincaré sphere, as a function of optical frequency. For each segment with fixed birefringence axis, the evolution equation for the Jones vector and dispersion vector are [7]

$$
\frac{\partial}{\partial z}\left(\begin{array}{l}
e_{1} \\
e_{2}
\end{array}\right)=\left(\begin{array}{cc}
i b & \frac{1}{2} g_{i} \\
-\frac{1}{2} g_{i} & -i b
\end{array}\right)\left(\begin{array}{l}
e_{1} \\
e_{2}
\end{array}\right)
$$

and

$$
\frac{\partial}{\partial z}\left(\begin{array}{l}
\Omega_{1} \\
\Omega_{2} \\
\Omega_{3}
\end{array}\right)=\left(\begin{array}{c}
\Omega_{2} \\
-\Omega_{1} \\
0
\end{array}\right) g_{i}+\left(\begin{array}{c}
2 b^{\prime} \\
-2 b \Omega_{3} \\
2 b \Omega_{2}
\end{array}\right)
$$

where $b=\Delta n \omega / 2 c$ and $b^{\prime}=\Delta n / 2 c$. The magnitude of the dispersion vector at the output of the fiber gives the differential group delay, $\Delta \tau(\omega)=|\Omega(\omega)|$, and its direction, $\hat{\Omega}=\Omega /|\Omega|$, determines the output principal states of polarization:

$$
\begin{gathered}
\hat{\boldsymbol{e}}_{b, 1 \pm}= \pm \frac{\left(\hat{\Omega}_{2}+i \hat{\Omega}_{3}\right)}{\sqrt{2\left(1 \mp \hat{\Omega}_{1}\right)}} \\
\hat{\boldsymbol{e}}_{b, 2 \pm}=\sqrt{\left(1 \mp \hat{\Omega}_{1}\right) / 2} .
\end{gathered}
$$

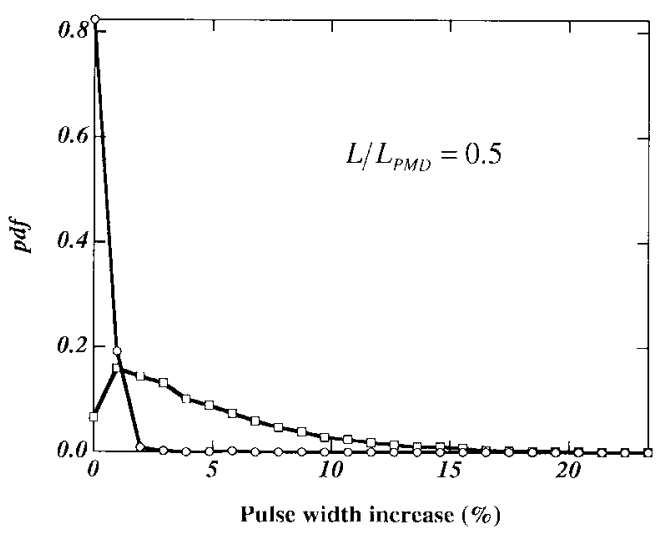

(a)

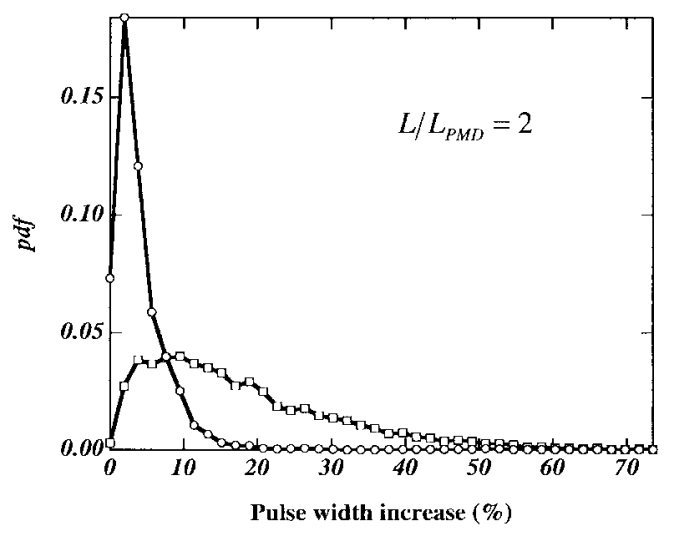

(b)

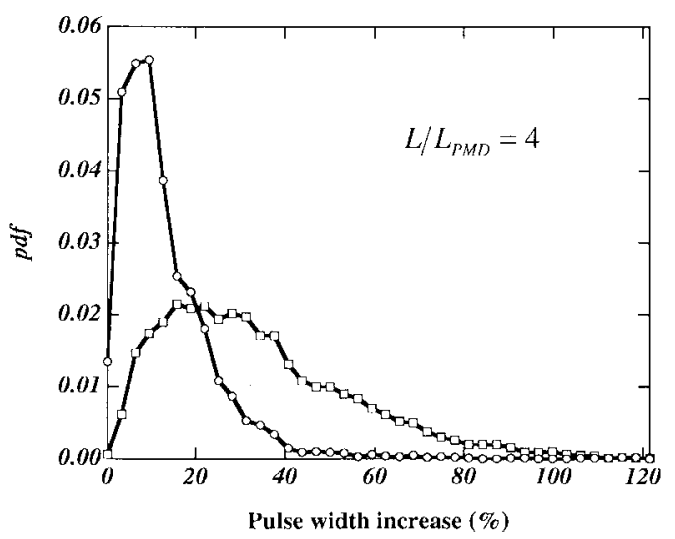

(c)

Fig. 1. Probability density functions for pulsewidth increase after propagation through fiber with various values of polarization mode dispersion. $\square$ : Without PMD compensation. $\bigcirc$ : with first-order PMD compensation. (a) $L / L_{\mathrm{PMD}}=0.5$. (b) $L / L_{\mathrm{PMD}}=2$. (c) $L / L_{\mathrm{PMD}}=4$.

These equations are the inverse of the well-known relations between the Jones vector components and their Poincaré sphere representations.

We calculated the rms pulsewidth of the output electric field with and without compensation for 33 fiber realizations at 9 different center frequencies, $\omega_{0}$. The separation between the center frequencies was larger than the decorrelation frequency width of the dispersion vector, $\Omega(z, \omega)$, thus ensuring that changing the center frequency is equivalent to choosing a statistically independent fiber realization. 
TABLE I

Comparison of the Means and Standard Deviations of the Distribution of Pulsewidths after Propagation Through a Fiber Without and With Polarization Mode DisPERSION COMPENSATION FOR VARIOUS VALUES OF $L / L_{\mathrm{PMD}}$

\begin{tabular}{c|c|c|c|c|c|c}
\hline$L / L_{\mathrm{PMD}}$ & $\bar{x}_{\text {un-comp }}$ & $\bar{x}_{\text {comp }}$ & $\bar{x}_{\text {un-comp }} / \bar{x}_{\text {couap }}$ & $\sigma_{\text {un-comp }}$ & $\sigma_{\text {comp }}$ & $\sigma_{\text {un-conq }} / \sigma_{\text {conle }}$ \\
\hline 0.12 & 1.12 & 0.005 & 224 & 0.994 & 0.034 & 29.3 \\
\hline 0.25 & 2.33 & 0.0585 & 39.8 & 2.01 & 0.152 & 13.3 \\
\hline 0.50 & 4.62 & 0.331 & 14.0 & 3.86 & 0.434 & 8.89 \\
\hline 0.75 & 6.87 & 0.655 & 10.5 & 5.57 & 0.939 & 5.93 \\
\hline 1.0 & 9.12 & 1.15 & 7.95 & 7.20 & 1.49 & 4.83 \\
\hline 1.25 & 11.30 & 1.76 & 6.42 & 8.66 & 2.12 & 4.08 \\
\hline 1.50 & 13.46 & 2.46 & 5.47 & 10.11 & 2.83 & 3.57 \\
\hline 1.75 & 15.6 & 3.27 & 4.77 & 11.5 & 3.61 & 3.17 \\
\hline 2.0 & 17.7 & 4.13 & 4.29 & 12.7 & 4.39 & 2.90 \\
\hline 4.0 & 33.9 & 13.3 & 2.54 & 21.3 & 11.7 & 1.82 \\
\hline
\end{tabular}

The input pulses had Gaussian envelopes and had widths such that $L / L_{\mathrm{PMD}}=0.12,0.25,0.5,0.75,1.0,1.25,1.75,2$, and 4 , corresponding to moderate to high polarization dispersion. For each fiber realization and center frequency the input state of polarization sampled 300 randomly chosen input states. Hence for a given ratio $L / L_{\mathrm{PMD}}, N=33 \times 9 \times 300=89100$ cases were tried.

For comparison with actual systems, $L / L_{\mathrm{PMD}}=0.12$ to $L / L_{\mathrm{PMD}}=4$ correspond to transmission of 50-ps full-width at half-maximum (FWHM) pulses (appropriate for $10 \mathrm{~Gb} / \mathrm{s}$ RZ) through a length of fiber having average differential group delays of $7.3 \mathrm{ps}$, to $40 \mathrm{ps}$, where the lower value of $7.3 \mathrm{ps}$ is expected to lead to a minimal power penalty. Considering a 400-km link, for example, these correspond to PMD values ranging from $D_{\mathrm{PMD}}=0.36$ to $D_{\mathrm{PMD}}=2 \mathrm{ps} / \mathrm{km}^{1 / 2}$.

Results: Fig. 1 shows probability density functions of the percentage pulsewidth increase with and without compensation for the three cases $L / L_{\mathrm{PMD}}=0.5,2$, and 4 . In every case, the differential group delay compensation sharpens the distribution of pulsewidths to smaller values. Table I shows the mean and standard deviation of the distribution of pulsewidths with and without compensation for all the cases. Compensation reduced the mean by factors $\bar{x}_{\mathrm{un}-\mathrm{comp}} / \bar{x}_{\mathrm{comp}}=224$ to $\bar{x}_{\mathrm{un}-\mathrm{comp}} / \bar{x}_{\mathrm{comp}}=2.54$ in the range $L / L_{\mathrm{PMD}}=0.12$ to $L / L_{\mathrm{PMD}}=4$. Compensation also reduced the standard deviation of the distribution of pulsewidths by factors $\sigma_{\mathrm{un}-\mathrm{comp}} / \sigma_{\mathrm{comp}}=29$ to $\sigma_{\mathrm{un}-\mathrm{comp}} / \sigma_{\mathrm{comp}}=1.82$ for the same range of $L / L_{\mathrm{PMD}}$. Clearly as dispersion increases, and the reduction factors approach unity, the advantage relative to the uncompensated values decreases, but the absolute advantage increases.

Compensation also reduced the tail of the distribution functions where the worst cases reside. As a measure of the tail of the distribution, we chose the pulse duration beyond which only $0.1 \%$ of the cases reside, $x_{w}$, and calculated it with and without compensation for all the cases. We found that $x_{w}$ values have the same dependence on $L / L_{\mathrm{PMD}}$ as the standard deviations with and without compensation.

Since the early work of Poole et al., it has been understood that differential group delay compensation significantly reduces pulse broadening for low PMD values. In this work we have found that at values of $L / L_{\mathrm{PMD}}$ as high as $L / L_{\mathrm{PMD}}=4$, there is a statistical advantage in doing first order PMD compensation, although the relative advantage steadily decreases with increasing PMD.

\section{ACKNOWLEDGMENT}

The authors acknowledge fruitful discussions with F. Heismann, J. Goldhar, and P. McGrath and thank the reviewer for helpful suggestions.

\section{REFERENCES}

[1] C. D. Poole and R. E. Wagner, "Phenomenological approach to polarization dispersion in long single-mode fibers," Electron. Lett., vol. 22, pp. 1029-1030, 1986.

[2] Z. Hass, C. D. Poole, M. A. Santoro, and J. H. Winters, "Fiber-optic transmission polarization-dependent distortion compensation," U.S. Patent 5311346, 1994.

[3] B. W. Hakki, "Polarization mode dispersion compensation by phase diversity detection," IEEE Photon. Technol. Lett., vol. 9, pp. 121-123, 1997.

[4] F. Heismann, D. A. Fishman, and D. Wilson, "Automatic compensation of first-order polarization mode dispersion in a $10 \mathrm{~Gb} / \mathrm{s}$ transmission system," in Tech. Dig., Eur. Conf. Optical Communication, 1998, pp. $529-530$.

[5] H. Resenfeldt and R. Eckhardt, "Broadband compensation of fiber birefringence," J. Lightwave Technol., submitted for publication.

[6] P. K. A. Wai and C. R. Menyuk, "Polarization mode dispersion, decorrelation, and diffusion in optical fibers with randomly varying birefringence," J. Lightwave Technol., vol. 14, pp. 148-157, 1996.

[7] C. D. Poole, J. H. Winters, and J. A. Nagel, "Dynamical equation for polarization dispersion," Opt. Lett., vol. 16, pp. 372-374, 1991. 